\title{
Part respectivement portée par les cadres théoriques et par les chercheurs dans la production de données intermédiaires issues des vidéos
}

Part linked to theoretical frameworks and to personal interpretation in the production of intermediate data in the analysis of classroom videos

\section{Patrice Venturini}

\section{OpenEdition \\ Journals}

Édition électronique

URL : https://journals.openedition.org/educationdidactique/1637

DOI : 10.4000/educationdidactique.1637

ISSN : 2111-4838

Éditeur

Presses universitaires de Rennes

\section{Édition imprimée}

Date de publication : 30 novembre 2012

Pagination : 161-166

ISBN : 978-2-7535-2254-1

ISSN : $1956-3485$

Référence électronique

Patrice Venturini, «Part respectivement portée par les cadres théoriques et par les chercheurs dans la production de données intermédiaires issues des vidéos », Éducation et didactique [En ligne], 6-3 | novembre 2012, mis en ligne le 30 novembre 2014, consulté le 25 avril 2022. URL : http:// journals.openedition.org/educationdidactique/1637 ; DOI : https://doi.org/10.4000/ educationdidactique.1637 


\title{
PART RESPECTIVEMENT PORTÉE PAR LES CADRES THÉORIQUES ET PAR LES CHERCHEURS DANS LA PRODUCTION DE DONNÉES INTERMÉDIAIRES ISSUES DES VIDÉOS
}

\author{
Patrice Venturini (EFTS, Université de Toulouse 2)
}

\begin{abstract}
Résumé: Lorsque les chercheurs analysent une vidéo de classe, ils sont généralement amenés à partir d'observables auxquels ils donnent un sens particulier à produire un premier ensemble de données intermédiaires sur lequel ils travaillent ensuite. Ces données intermédiaires sont produites à l'aide de cadres théoriques, mais leur production est aussi liée à des interprétations et des choix implicites ou explicites du chercheur. Larticle a pour but de discuter dans les articles de ce numéro, la répartition entre ces deux pôles (cadre théorique - chercheur) lors des toutes premières analyses et conclut sur la nécessité d'une réflexion méthodologique approfondie afin de mieux formaliser les procédures utilisées
\end{abstract}

Mots clés: Méthodologie; analyse de vidéos de classe; données intermédiaires; structuration de données.

Patrice Venturini

\section{Objet de la discussion}

Lorsqu'on utilise une vidéo pour analyser une séance d'enseignement, on est généralement amené à identifier dans celle-ci des « observables » et à leur attribuer un sens particulier, pour produire des découpages qui structurent le réel observé ainsi que des données qui feront ensuite l'objet de divers traitements, qualitatifs ou quantitatifs. Cet ensemble (structures et données) constitue ce que j'appellerai par la suite des « données intermédiaires ", intermédiaires parce que c'est sur elles que travaille ensuite le chercheur pour produire ses résultats. Ces « données intermédiaires » sont donc issues d'un premier traitement de la vidéo dans lequel les éléments théoriques jouent un rôle plus ou moins important selon les études et sont associés de manière implicite ou explicite à des choix et des inférences liées aux interprétations du chercheur qui peuvent concernent le repérage des observables, le sens qui leur est attribué, ou les deux.

Les résultats mis en valeur dans un article sont donc directement liés aux données intermédiaires produites par les chercheurs qui l'ont rédigé et les cinq articles présentés dans ce numéro n'échappent pas à cette règle. Aussi, je me propose de discuter ici dans chaque cas les relations que ces données intermédiaires entretiennent avec les différents cadres théoriques utilisés et la part qu'elles semblent devoir au chercheur. La discussion qui suit est donc structurée par ces deux critères, sans que l'ordre avec lequel sont traités les différents articles ne reflète une quelconque hiérarchie dans mon esprit. Le premier d'entre eux à être discuté développe une analyse référentielle du discours pour laquelle le cadre théorique supporte fortement le chercheur lors de la constitution des données intermédiaires.

\section{Le cadre théorique supporte fortement le chercheur dont l'interprétation est minimale}

Le cadre que propose L. Lima (cf. ce numéro, p. 39-61) comporte à la fois un certain nombre de concepts et de principes (modélisation d'une situation à partir des référents présents dans le discours, centralité de l'information associée au principe d'activation et de désactivation de l'information, principe d'accumulation, seuil d'activation, ...) ainsi que leurs modalités d'opérationnalisation (algorithme permettant d'établir le niveau d'activation d'un référant à tout moment de la séance). Le rôle du chercheur est ici d'identifier dans le discours de l'enseignant les référents supposés participer à la représentation que les élèves se feront de la situation étudiée. Ce repérage inclut non seulement les apparitions explicites du référent mais aussi leurs anaphores nominales ou pronominales ou les déictiques correspondants. À chacune de ces occurrences, le chercheur associe une information temporelle. Il construit ainsi un tableau comportant une colonne par référent et une 
ligne par occurrence référentielle, tableau sur lequel l'algorithme de détermination du niveau d'activation est alors appliqué. Le nouveau tableau obtenu à partir de l'application de l'algorithme indique à un instant donné, l'ensemble des référents activés ainsi que leur niveau d'activation et regroupe donc les données intermédiaires qui feront ensuite l'objet d'un traitement statistique. Le chercheur a donc ici à choisir les référents: ce sont les substantifs, « objets du monde » évoqués dans les interactions ainsi que les formes référentielles associées (en excluant, les connecteurs, les verbes ainsi que les parties extérieures à la transmission des savoirs, comme la gestion de classe). Dans le cas de cette séance on trouve par exemple parmi les référents les termes étoile, document, spectroscope, exercices, spectre, raies, couleur, activité, lumière, etc. Le sens donné à ces référents (niveau d'activation) repose quant à lui uniquement sur l'algorithme de traitement porté par le cadre théorique. La sélection des référents ne nécessitant pas une expertise disciplinaire avérée, on peut donc avancer l'idée que la constitution de ces données intermédiaires repose dans ce cas beaucoup plus sur le cadre théorique que sur l'interprétation du chercheur qui est ici minimale.

En poursuivant la classification proposée, deux autres articles sont maintenant discutés dans lesquels de mon point de vue, les cadres théoriques concourent à donner une légitimité à l'analyse proposée en l'inscrivant dans un contexte plus large mais ne fournissent pas directement les outils pour l'investigation. L'interprétation du chercheur quant à elle varie alors selon les objets analysés.

\section{Le cadre théorique légitime l'étude menée sans fournir au chercheur des outils utilisables dans les investigations. La part d'interprétation de ce dernier est variable}

Ainsi dans son article « Évolution de l'action de l'enseignant et performances didactiques des élèves. Analyse didactique d'une séance en sciences physiques au lycée vidéo-observée " (cf. ce numéro, p. 19-38), MP. Chopin fait état des travaux réalisés sur la mémoire didactique et sur le modèle d'hétérogénéisation. Ceux-ci justifient que l'on s'intéresse au développement temporel du savoir. Ils justifient que l'on cherche à documenter différents aspects de « la nature du temps de la diffusion des savoirs » tout comme l'équilibre subtil entre passé, présent et futur didactiques, maintenu par l'enseignant tout au long de la séance. Le cadre théorique conduit à rechercher dans le discours de l'enseignant, les allusions au passé, au présent et au futur dans les différentes interactions puis à les caractériser. Leur repérage se fait à partir d'indicateurs linguistiques liés au temps (par exemple: «il y a quinze jours, on disait...»). Ces « mentions au temps » font l'objet d'une double catégorisation définie a priori, en lien avec ce à quoi elles réfèrent et avec leur auteur. Ainsi, selon le cas, ces allusions temporelles renvoient à des activités menées dans la classe (par exemple des expériences et les observations qu'elles ont permises), à des " éléments de savoir en jeu dans la leçon » (des explications, une définition, etc.) ou encore à des aspects plus instrumentaux (par exemple rappel de la fiche qu'il a fallu revoir, ou qui sera remplie plus tard). Elles sont effectuées par l'enseignant, par un élève ou encore conjointement par les deux. Enfin, ces mentions sont regroupées par grandes phases didactiques (dénommées "fin de l'activité précédente", « mise en commun », «bilan de l'activité ») (et sous-phases: lancement de l'activité, travail collectif etc.), repérées du point de vue du professeur. Les données intermédiaires sont ainsi constituées par les phases et sous-phases qui structurent la séance et par le nombre d'occurrences de chaque catégorie pour chacune des phases et sous-phases, leurs nombres faisant ensuite l'objet d'un traitement statistique. Dans ce processus méthodologique, les observables (éléments linguistiques liés au temps et éléments linguistiques marquant le début et la fin des phases didactiques) sont généralement faciles à identifier dans le flot du discours. Il reste au chercheur à identifier les catégories liées aux allusions temporelles. Par exemple, il lui appartient d'inférer du contexte si des observables comme « on a vu la décomposition de la lumière lors de la dernière séance » renvoient plutôt à une activité ou à un contenu. Même sans outil d'investigation fourni par le cadre théorique, l'interprétation du chercheur et l'expertise disciplinaire requise semblent réduites.

Dans l'article « Décrire, comprendre et expliquer les pratiques d'enseignement d'un professeur de lycée » proposé par L. Talbot et A. Arrieu-Mutel (cf. ce numéro, p. 63-93), le cadre théorique a la même fonction vis-à-vis de la vidéo que dans le précédent ${ }^{1}$. 
Les auteurs fondent leur étude sur l'approche sociocognitive de Bandura pour lequel une pratique (et donc la pratique d'enseignement) peut se caractériser par trois dimensions, interne au sujet, environnementale et comportementale. Cette dernière renvoie à l'activité de l'enseignant, caractérisable selon les auteurs à partir de sept macro-variables d'action mises à jour par les travaux sur "l'effet maître ». L'une d'entre elle est relative à l'évaluation, importante parce qu'elle permet de suivre les progrès des élèves. S'appuyant sur ce résultat, les auteurs proposent de centrer leur analyse sur "l'activité orale évaluative » de l'enseignant, même si d'autres types d'interventions sont aussi pris en compte: propos liés à l'aide apportée aux élèves, à des renforcements négatifs ou positifs ou à des aspects proxémiques. Il s'agit d'identifier toutes les deux secondes si les propos de l'enseignant s'adressent à un individu ou au collectif, relèvent de l'évaluation sommative, diagnostique ou formative, de l'aide ou du renforcement à partir d'indicateurs linguistiques. La grille d'observation, qui une fois remplie comportera les données intermédiaires qui feront ensuite l'objet d'un traitement statistique, inclut aussi le statut scolaire des élèves et leur sexe, en vertu d'un certain nombre d'hypothèses liées à des recherches précédentes sur les comportements différenciés des enseignants, évoquées dans la méthodologie. Si l'objet que l'on regarde dans les interactions est légitimé par le cadre théorique (au moins pour l'activité d'évaluation), rien dans celuici ne guide le chercheur pour l'identification des observables concernés et leur caractérisation. À quels propos attribuer une valeur évaluative? En vertu de quoi et quelle valeur attribuer? Si la recherche d'observables particuliers liés à l'évaluation est justifiée par le cadre théorique, leur repérage dans le flux de l'action et le sens qu'on leur attribue dépendent ici fortement des interprétations du chercheur. Par exemple, les auteurs considèrent que les propos «Qu'est-ce qu'on a fait dans l'activité 2?» relèvent d'une évaluation diagnostique, mais on pourrait tout aussi bien considérer que l'enseignant cherche ainsi à introduire un élément significatif dans le milieu, ou encore qu'il opère une évaluation sommative. On pourrait discuter de la même manière d'autres exemples fournis par les auteurs comme «c'est bien, continue ainsi... » qu'ils repèrent comme une évaluation formative mais qui pourrait aussi constituer un encouragement ou «non, pas du tout...», identifié comme une évaluation sommative, mais que l'on pourrait lire comme une évaluation formative. Il ne s'agit pas pour moi ici de mettre en cause la pertinence de leur analyse mais de pointer la part d'interprétation des chercheurs. Si l'identification des exemples donnés semble reliée à l'interprétation d'indicateurs linguistiques, on peut s'interroger sur l'interprétation du contexte, en particulier disciplinaire, que ces caractérisations nécessitent.

Mais ces interrogations existent aussi dans d'autres cas, par exemple lorsque le cadre théorique définit de manière insuffisamment opérationnelle les concepts qu'il comporte.

\section{Le cadre théorique supporte insuffisamment le chercheur et lui laisse ainsi une part d'interprétation importante}

Cela me semble être le cas dans l'article d'A. Zaid (cf. ce numéro, p. 123-145) « Analyse didactique d'une situation contrainte d'enseignement des sciences au lycée " pour lequel le concept de «performance didactique » est central dans le cadre théorique. «Effet du système didactique construit par un observateur (enseignant, chercheur, ...) », la performance didactique vise à rendre compte aussi bien « des savoirs, des savoirfaire, des rapports à, des attitudes, des conduites et des compétences [...] que de rapports plus complexes tel que le rapport entre compétences, dynamique de l'élève et pratiques d'enseignement ». La performance didactique est aussi « l'expression manifeste, inférée par le chercheur, du rapport de l'élève au contenu en tant que forme d'engagement dans la situation de classe ", ou encore une « image des apprentissages de l'élève " à l'origine des évolutions du système didactique. Toutefois, ces différentes définitions fournies dans l'article sont soit peu explicites (effet du système didactique, image des apprentissages), soit incluent des concepts comme les rapports à ou entre, les compétences, les conduites ou encore les attitudes renvoyant potentiellement à de multiples définitions et à de multiples référents dans le réel que les observables mentionnés (paroles, gestes, usage d'outils, silences) ne spécifient pas. Par ailleurs, le cadre proposé définit l'action de l'enseignant comme la sélection parmi les performances didactiques de l'élève de « celles qui lui permettent le mieux d'effectuer les cadrages nécessaires [...] de progresser dans le déploiement de son dispositif didactique et pédagogique ». Ce lien amène 
le chercheur à analyser l'action de l'enseignant et les performances des élèves dans la séance de physique. Pour cela, la séance est découpée en trois phases liées à l'action didactique de l'enseignant (dénommées « préparation et énoncé de la tâche », « réalisation en groupe », « correction et structuration »). Dans chaque phase sont repérées les performances didactiques des élèves ainsi que des «événements » caractérisés par un changement de l'action de l'enseignant. Les performances font l'objet d'une catégorisation définie a priori. Elles peuvent être cognitives (dominante notionnelle), techniques (dominante liée à l'usage d'objets), sociales (dominante d'interaction avec les pairs) ou de silence (situations d'écoute par exemple), et traduire une réussite ou un échec de l'élève. Deux événements délimitent un « segment » de l'action. Les données intermédiaires qui feront ensuite l'objet de mesures fréquentielles ou de représentations graphiques rassemblent les différents segments avec les événements liés au début et à la fin de chacun d'entre eux (repérés dans le temps), ainsi que les performances didactiques associées ou non à ces événements avec leur caractérisation. Toutefois, cet ensemble méthodologique n'est pas supporté par le cadre théorique. Le choix des actions retenues (qui ne sont pas connues a priori et sont de nature très différente), les critères de repérage de ces actions ou de leur changement, sont à la charge du chercheur, tout comme l'identification dans les observables « élève » de performances didactiques ainsi que leur caractérisation sans que celles-ci n'aient été clairement spécifiées. Pour mener à bien tout cela, une expertise disciplinaire est certainement nécessaire, en tout cas beaucoup plus que dans les cas précédents. Seule l'identification des phases de l'action didactique repose sur quelques observables linguistiques et comportementaux aisément repérables.

Ceci étant, dans certains cas, une définition peut-être plus établie des concepts du cadre théorique mobilisé ne dispense pas toujours pour autant le chercheur d'interprétations importantes dans la constitution de ses données intermédiaires.

Le cadre théorique est structuré et les concepts définis mais il contraint pourtant le chercheur à de fortes interprétations pour son utilisation

L'article d'A. Tiberghien «Analyse d'une séance en physique, quelle continuité pour les pratiques» (cf. ce numéro, p. 95-121) me paraît relever de cette catégorie. Le cadre théorique proposé dans l'article comporte trois ensembles articulés: théorie de l'action conjointe en didactique, modélisation des échelles de temps et modélisation de l'apprentissage. Il inclut donc de nombreux concepts (trop nombreux pour être brièvement rappelés ici), de type relationnel, complexes à manipuler, qui sont mis en jeu dans la structuration de la séance. Les différentes structurations qui en résultent constituent les données intermédiaires qui seront ensuite analysées généralement de manière qualitative. Si la structuration selon l'organisation de la classe ne pose aucun problème, il n'en est pas de même pour les autres. Ainsi, la séance est découpée en jeux d'apprentissage, chacun défini par un contrat et un milieu didactiques, un enjeu et une règle du jeu, concepts et notions tous précisés au sein de la théorie de l'action conjointe en didactique. Mais plusieurs dimensions de la théorie sont mises en jeu de concert dans cette structuration, par exemple l'évolution du savoir, la responsabilité dans son avancée, l'évolution du milieu et du contrat au cours du temps. Il s'agit donc pour le chercheur qui identifie les jeux successifs de faire preuve d'une profonde compréhension des transactions à propos des savoirs entre enseignant et élèves et de leurs points de vue respectifs pour découper ainsi le flux de l'action didactique. Il doit repérer des événements particuliers (observables) dont la portée temporelle est variable, inférer le sens qu'ils portent, les mettre en relation pour spécifier les différents concepts, structurer et spécifier l'action didactique en combinant ces derniers. Appuyées sur deux des ensembles théoriques articulés, deux autres structurations d'ordre chronogénétique sont proposées au niveau mésoscopique et au niveau microscopique, pour lesquelles il n'existe pas de catégories définies a priori. La première est relative aux thèmes abordés dans la classe. Elle est fondée sur des indicateurs linguistiques et chaque unité est décrite en fonction de la signification qu'elle a du point de vue de l'élève ou du professeur. Les inférences qui permettent cette caractérisation sont supportées par une expertise sur les savoirs et sur les pratiques disciplinaires, expertise partagée au sein de la communauté des didacticiens de la physique. La seconde est relative aux microéléments de savoir scientifique ou naif (facettes) découpés dans le flux du discours de l'enseignant et des élèves. Réalisé ici sans référence à un catalogue déjà existant, ce découpage et le regroupement éven- 
tuel des facettes selon leur nature nécessitent eux aussi une expertise disciplinaire. Par ailleurs, en fonction de la durée de la séquence analysée, le chercheur doit déterminer la granularité des éléments mésoscopiques qu'il analyse. Ainsi au sein d'un jeu ou d'un thème, il peut exister des « sous-jeux » ou des « sous-thèmes » qui avec une granularité différente pourraient avoir le statut de jeux ou de thèmes à part entière. Finalement, malgré un cadre théorique cohérent articulant plusieurs aspects (ou en raison de ce cadre?), la part d'interprétation du chercheur est ici aussi très importante dans la construction des données intermédiaires, tant dans le choix et le repérage d'observables que dans les inférences faites quant au sens qu'ils portent.

Après avoir examiné ces cinq articles, que retenir finalement de cette discussion?

\section{Conclusion}

Tout d'abord, il ne s'agissait pour moi en aucun cas, ni de comparer ces travaux pour établir une hiérarchie qualitative en leur sein, ni de remettre en cause, comme je l'ai dit plus haut, la pertinence des méthodologies proposées, mais bien, en interrogeant les liens entre les cadres théoriques et les méthodologies que nous utilisons tous, de prendre conscience des parts respectivement portées par les outils théoriques et par le chercheur dans la construction des données intermédiaires qualitatives ou quantitatives. On peut légitimement penser au vu des analyses précédentes que nous avons probablement tous besoin d'affiner les outils que nous utilisons afin de mieux fonder nos méthodologies (à cet égard par exemple, l'analyse référentielle du discours pourrait être testée pour assurer la structuration thématique d'une séance). Mais nous avons aussi besoin d'objectiver davantage la part portée par le chercheur dans ces dernières. L'enjeu est à la fois de donner plus de poids aux résultats tirés des données intermédiaires construites à partir des vidéos mais aussi de contribuer à la formation des jeunes chercheurs, ou à rendre la méthodologie disponible pour d'autres. Plus fondamentalement, l'enjeu est de nature épistémologique: la science didactique se doit autant que faire se peut, comme toutes les sciences, de clarifier la manière dont elle construit ses savoirs, pour assurer sa légitimité (et probablement son efficacité). Toutefois, ce travail qui apparaît nécessaire n'est pas forcément productif dans l'immédiat pour le chercheur qui s'y livre, est peu susceptible d'obtenir des financements spécifiques et se heurte aussi à la taille imposée aux articles dans la plupart des revues. Les lieux dans lesquels il est possible, comme par exemple dans ce cas d'espèce au sein du projet ViSA sont d'autant plus précieux, sans penser pour autant qu'on arrivera totalement à objectiver la part portée par le sujet, et encore moins à la supprimer.

J'adresse mes remerciements à Andrée Tiberghien dont l'analyse critique sur la distance entre observables et interprétation dans son article au sein de ce numéro spécial ainsi que les échanges que nous avons par ailleurs m'ont sensibilisé à cette problématique et ont ainsi permis l'existence de cette discussion. 


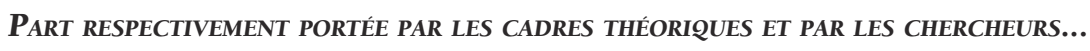

\section{Patrice Venturini}

\section{NOTES}

1. Cet article développe d'autres analyses en complément de l'analyse de la séance vidéoscopée que nous ne prenons pas en compte ici puisque notre discussion est centrée sur la constitution de données intermédiaires à partir de la vidéo 